\title{
Intratumoral Therapy
}

National Cancer Institute

\section{Source}

National Cancer Institute. Intratumoral Therapy. NCI Thesaurus. Code C62722.

Direct administration of a therapeutic agent into a malignant tumor. 